\title{
Nutritional Quality Characteristics of Different Types of Guangdong-Style Mooncakes
}

\author{
Chunying Yang, Xueming Liu, Zhiyi Chen, Yaosheng Lin, Xiaoli Zhao, and Rongling Yang \\ Sericultural and Agri-Food Research Institute, Guangdong Academy of Agricultural Sciences, \\ Key Laboratory of Functional Foods, Ministry of Agriculture, and Guangdong Key Laboratory of Agricultural Products Processing, \\ 133 Yihenglu, Dongguanzhuang, Tianhe District, Guangzhou 510610, China \\ Correspondence should be addressed to Xueming Liu; xuemingliu@21cn.com
}

Received 1 August 2015; Revised 8 October 2015; Accepted 11 October 2015

Academic Editor: Subramaniam Sathivel

Copyright (C) 2015 Chunying Yang et al. This is an open access article distributed under the Creative Commons Attribution License, which permits unrestricted use, distribution, and reproduction in any medium, provided the original work is properly cited.

\begin{abstract}
This study was designed to clarify nutritional characteristics of Guangdong-style mooncakes. Proximate composition, fatty acid profile, and the cholesterol content of Guangdong-style mooncake types (DYLP, SYLP, LP, BP, FF, and FK) were analyzed and their energy was calculated. Guangdong-style mooncakes contained $15.96 \sim 19.16 \%$ moisture, $32.06 \sim 43.12 \%$ total reducing sugar, $5.35 \sim$ $9.06 \%$ protein, and $3.72 \sim 18.74 \%$ crude fat and could provide $930.52 \sim 1403.30 \mathrm{~kJ} / 100 \mathrm{~g}$ of energy excluding starch. FK mooncake had the lowest moisture, and LP, BP, and FF mooncakes contained high levels of reducing sugar. The main soluble sugars in Guangdongstyle mooncakes were sucrose, glucose, and fructose. The fat content order was as follows: DYLP $>$ SYLP $>$ FK $>$ BP $>$ LP $>$ FF, where DYLP contained $18.74 \%$ fat while FF only contained 3.72\%. Dominating fatty acids in Guangdong-style mooncakes were $\mathrm{C}_{18: 1}$ and $\mathrm{C}_{18: 2}$. Cholesterol was mainly found in yolk-type mooncakes. FK mooncake had the highest protein content (9.06\%). Guangdong-style mooncakes are high in moisture and sugar, but not always high in fat.
\end{abstract}

\section{Introduction}

Mooncakes are Chinese pastries consumed during the MidAutumn Festival. They are round or square baked with fillings and their crusts are decorated with imprints of the manufacturers and auspicious greetings or indicate the ingredients [1, 2]. Mooncakes are not only delicious foods, but also cultural products in China $[3,4]$.

Mooncakes consist of crust and filling; the former is made of wheat or other types of cereal flour and vegetable oil, with or without sugar, usually with decorative pattern on it, while the latter contains different paste (lotus seed paste, bean paste, taro paste, etc.), salted yolk, fruits, seed kernels, sesame, pork, or other edible materials [5].

Recipes for mooncakes vary across China depending on the formulation, type, and manufacturing process. According to their origin places and tastes, mooncakes can be classified into Guangdong-style, Beijing-style, Suzhou-style, and other regional styles. The Guangdong-style mooncake was originally from Guangzhou, but it is now produced all over the country, especially in Guangdong, Hong Kong, Shanghai,
Jiangxi, and Zhejiang. Presently, they account for more than $70 \%$ of total mooncake market in China [6].

The feature of Guangdong-style mooncake is thin skin, thick filling, and tender texture. Typical Guangdong-style mooncakes are round or rectangular pastries, measuring about $10 \mathrm{~cm}$ in diameter and $4-5 \mathrm{~cm}$ thick. The skin is made of wheat flour, invert syrup, vegetable oil, and Kanshui (clear water solution of plant ash) which is replaced with lye water in contemporary mooncake industries. The fillings are selected from local special edible material with high diversification, including seeds (lotus, beans, sesame, olive kernel, almond, walnut meat, etc.), fruits (coconut shred, pineapple, durian, banana, orange cake, dried orange peel, etc.), vegetables (taro, edible mushroom, sugar coated winter melon, etc.), eggs and meats (salted yolk, Guangdong-style sausage, barbecued pork, sugar cured fat, etc.), and even seafood (abalone, shark fin, dried scallop, etc.). Common Guangdong-style mooncake types in the present Chinese market are lotus seed paste with yolk, pure lotus seed paste, bean paste, five kernels, fruit flavor, and roasted meat type. 
TABLE 1: Sample information.

\begin{tabular}{|c|c|c|c|c|c|c|c|c|}
\hline \multirow{2}{*}{ Brand name } & \multicolumn{8}{|c|}{ Type } \\
\hline & DYLP & SYLP & LP & BP & FF & FK & Other & Subtotal \\
\hline An Qi & 0 & 0 & 1 & 0 & 1 & 1 & 2 & 5 \\
\hline Di Shi Ni & 0 & 1 & 0 & 0 & 2 & 0 & 3 & 6 \\
\hline Dong Hai Tang & 1 & 0 & 0 & 0 & 0 & 1 & 0 & 2 \\
\hline Guangzhou Jiujia & 2 & 5 & 0 & 0 & 0 & 2 & 0 & 9 \\
\hline Guangzhou Renjia & 1 & 2 & 2 & 0 & 1 & 1 & 1 & 8 \\
\hline Guangzhou Sibao & 0 & 1 & 1 & 1 & 1 & 0 & 0 & 4 \\
\hline Hongkong Huangguan & 1 & 1 & 0 & 1 & 2 & 0 & 1 & 6 \\
\hline Hongkong Pinjia & 0 & 2 & 1 & 0 & 0 & 0 & 1 & 4 \\
\hline Hongkong Tangxin & 0 & 1 & 0 & 1 & 1 & 0 & 0 & 3 \\
\hline Jin Yu Xiang & 0 & 0 & 0 & 0 & 3 & 0 & 0 & 3 \\
\hline Lian Xiang Lou & 1 & 1 & 1 & 0 & 0 & 1 & 0 & 4 \\
\hline Mei Xin & 6 & 0 & 0 & 0 & 0 & 1 & 0 & 7 \\
\hline Qu Xiang & 2 & 3 & 1 & 2 & 0 & 1 & 0 & 9 \\
\hline Rong Hua & 5 & 1 & 0 & 0 & 0 & 1 & 0 & 7 \\
\hline Tao Tao Ju & 3 & 2 & 1 & 2 & 0 & 2 & 0 & 10 \\
\hline Tian Xing Long & 2 & 2 & 1 & 2 & 2 & 1 & 1 & 11 \\
\hline Zhuang Yuan Fang & 0 & 1 & 1 & 0 & 0 & 0 & 0 & 2 \\
\hline Total & 24 & 23 & 10 & 9 & 13 & 12 & 9 & 100 \\
\hline
\end{tabular}

${ }^{*}$ DYLP: double-yolk lotus seed paste mooncake; SYLP: single-yolk lotus seed paste mooncake; LP: pure lotus seed paste mooncake; BP: bean paste mooncake; FF: fruit flavor mooncake; FK: five-kernel mooncake.

Guangdong-style mooncakes are traditionally thought as high sugar and high fat foods; however, few reports on their nutrition evaluation could be found. In order to explore the nutritional features of Guangdong-style mooncakes and to give detailed nutritional information to consumers and producers, a study was undertaken to investigate proximate composition, fatty acid profile, and cholesterol content of the main types of Guangdong-style mooncakes.

\section{Materials and Methods}

2.1. Materials. All the chemicals used in the experiments were of HPLC or analytical grade. Glucose, fructose, sucrose, and cholesterol were purchased from Sigma-Aldrich, USA.

Forty-five boxes of mooncakes of 17 different brands were purchased from markets in Guangzhou in September, 2011. A hundred mooncake samples were selected according to their brands and recipes, including 24 double-yolk lotus seed paste mooncakes (DYLP), 23 single-yolk lotus seed paste mooncakes (SYLP), 10 pure lotus seed paste mooncakes (LP), 12 five-kernel mooncakes (FK), 9 bean paste mooncakes (BP), 13 fruit flavor mooncakes (FF), and 9 other mooncake samples (with fish, meat, cheese, tea, etc.). Mooncake sample information is shown in Table 1.

After texture evaluation, all samples were cut into small cubes $(0.2 \sim 0.3 \mathrm{~cm} \times 0.2 \sim 0.3 \mathrm{~cm} \times 0.2 \sim 0.3 \mathrm{~cm})$ and then ground to paste. Due to the high moisture, lipid, and sugar contents, the mooncake paste could not be sieved or mixed in a mixer. Alternatively, the paste was put in a plastic bag and mixed manually. All samples were kept in a freezer at $-20^{\circ} \mathrm{C}$ until analysis.
2.2. Texture Evaluation. Mooncake texture was determined by a TA-XTplus texture analyzer (Stable Microsystems, Surrey, UK) which was equipped with the software "Texture Expert" using the method optimized by our institute [7]. An aluminum $50 \mathrm{~mm}$ diameter cylindrical probe was used in a Texture Profile Analysis (TPA) compression test to penetrate to $90 \%$ depth, at a speed of $2.0 \mathrm{~mm} / \mathrm{s}$. Only one compression was performed due to the high viscosity, poor fracturability, springiness, and resilience of Guangdong-style mooncakes. Firmness $(N)$ and cohesiveness were calculated from the TPA graphic [8]. For consistency of results, specimens of approximately the same thickness and width were selected and samples of $40 \mathrm{~mm} \times 40 \mathrm{~mm} \times 20 \mathrm{~mm}$ were used.

2.3. Proximate Composition. Proximate analyses were carried out according to the methods described by the Association of Official Analytical Chemists [9]. All the analyses were carried out in triplicate. Moisture was tested for all samples. The moisture content was estimated by hot air oven method with a gravimetric moisture analyzer (Sartorius MA 100, Germany). It was determined by drying a known weight of pasted sample in a hot air oven to constant weight. The dried materials were analyzed for proximal components, namely, sugar, ether-extractable residues (crude fat), and crude protein, by the AOAC methods. Sugars were determined by the LaneEynon general volumetric method as reported by the AOAC (Method 923.09). Sugars present in samples were extracted with distilled water, and nonreducing sugars present in the aqueous extract were converted to reducing sugars by reacting with hydrochloric acid $(6 \mathrm{~mol} / \mathrm{L})$ and titrated against boiling $\mathrm{Cu}$ (II) solution with methylene blue as the indicator. 
Fat content was estimated by Soxhlet extraction of known weights of the samples (AOAC, 2000). Fats were extracted using petroleum ether (b.pt. $60-90^{\circ}$ ) in a Soxhlet extractor (SOX 416 Macro 6, Gerhardt, Germany). Extraction was carried out at $60^{\circ} \mathrm{C}$. Extracted fats were weighed and reported. The protein $(N \times 6.25)$ content was determined by the macro-Kjeldahl method. The caloric value was calculated by using the Atwater energy conversion factors and according to the following equation: energy $(\mathrm{kJ})=17 \times(\mathrm{g}$ protein $+\mathrm{g}$ carbohydrate $)+37 \times($ g lipid $)$.

2.4. Fatty Acid Profile. The mooncake samples were partially dried in a vacuum oven $\left(70^{\circ} \mathrm{C}\right.$ for $\left.3 \mathrm{~h}\right)$ and the fat was extracted in a Soxhlet apparatus (SOX 416 Macro 6, Gerhardt, Germany) using petroleum ether (b.pt. $60-90^{\circ}$ ). The residual moisture in oil was removed using anhydrous sodium sulfate. The fatty acid composition of these oil samples was determined by analyzing the fatty acid methyl esters by gas chromatography-mass spectrometer (GC-MS).

Fatty acid methyl esters (FAMEs) were prepared by using $\mathrm{NaOH} /$ methanol method [10]. About $50-80 \mathrm{mg}$ of oil was transferred into a ground glass stoppered test tube, treated with $0.5 \mathrm{~mol} / \mathrm{L} \mathrm{NaOH} /$ methanol. The samples were mixed for $20 \mathrm{~s}$ on vortex mixer and shaken once every $5 \mathrm{~min}$, with a reaction time of $40 \mathrm{~min}$ at ambient temperature. The methyl esters were extracted using $n$-hexane and the aqueous phase was discarded. The $n$-hexane extract containing the FAMEs was washed with water and dried using anhydrous sodium sulfate and stored at $-20^{\circ} \mathrm{C}$ for later analysis by GC-MS.

FAMEs were determined with gas chromatographymass spectrometry (Model 6890N/5975B; Agilent, California, USA) equipped with a treated J\&W DB-WAX polyethylene glycol column $(30 \mathrm{~m} \times 0.25 \mathrm{~mm}, 0.25 \mu \mathrm{m}$; J\&W Scientific, USA). FAMEs were separated and detected and their concentration was measured. The GC oven program was the following: $180^{\circ} \mathrm{C}$ (hold $5 \mathrm{~min}$ ) to $230^{\circ} \mathrm{C}$ at $3^{\circ} \mathrm{C} / \mathrm{min}$ (hold $15 \mathrm{~min}$ ). The carrier gas (helium) flow rate was in constant flow mode at $1 \mathrm{~mL} / \mathrm{min}$. Splitless injection of $1 \mu \mathrm{L}$ was carried out at $250^{\circ} \mathrm{C}$ with the purge valve on at $2 \mathrm{~min}$. The mass spectra were obtained in electron ionization mode at $70 \mathrm{eV}$. Ion source temperature was $230^{\circ} \mathrm{C}$, while transfer line temperature was $280^{\circ} \mathrm{C}$. Full scan mass spectra were acquired in the mass range of $30-450 \mathrm{Da}$ with a 3 min solvent delay. The results are expressed in relative percentage of each fatty acid, calculated by normalization of the chromatographic peak area according to GB/T 17377-2008 (China). Fatty acid identification was made by analysis of MS spectra and indexing of NIST05aL spectral database.

2.5. Cholesterol. The method of ferric ammonium sulfate color reagent was used to determine the cholesterol amount in the mooncakes [11]. Fat was extracted in a Soxhlet apparatus (SOX 416 Macro 6, Gerhardt, Germany) using petroleum ether (b.pt. 60-90 ). Samples were saponified using the alcoholic $\mathrm{KOH}$ method [12]. Fat samples $(0.20 \mathrm{~g}$ each) were saponified directly with $4.5 \mathrm{~mL}$ of alcoholic $\mathrm{KOH}$ solution ( $4.0 \mathrm{~mL} 95 \%$ ethanol plus $0.5 \mathrm{~mL} 50 \% \mathrm{KOH}$ ) in tightly capped tubes in a $65^{\circ} \mathrm{C}$ water bath for $1 \mathrm{~h}$, with occasional shaking. After cooling to room temperature, $3.0 \mathrm{~mL} 50 \mathrm{~g} / \mathrm{L} \mathrm{NaCl}$ solution was added and the mixture was extracted with $10 \mathrm{~mL}$ petroleum ether $\left(30-60^{\circ} \mathrm{C}\right)$ and vortexed $(2 \mathrm{~min})$. The organic phase was transferred to $10 \mathrm{~mL}$ test tube and evaporated to dryness in a $65^{\circ} \mathrm{C}$ water bath with nitrogen gas purging. The residue was treated with $4.0 \mathrm{~mL}$ glacial acetic acid and $2.0 \mathrm{~mL}$ ferric ammonium sulfate color reagent and mixed. After $15 \mathrm{~min}$, absorbance values were read at $562 \mathrm{~nm}$ using a spectrophotometer (Shimadzu UV-2450; Shimadzu Corporation, Chiyoda-ku, Tokyo, Japan). All samples were run in triplicate. Corrected absorbance values were interpolated in the corresponding calibration line obtained for standard solutions containing from 0.0875 to $0.7 \mathrm{mg} / \mathrm{mL}$ cholesterol prepared and treated in the same way as samples.

2.6. Statistical Analyses. For triplicate experimental data, mean and standard deviations were calculated using Microsoft Excel 2010. The results were expressed as mean \pm standard deviation. One-way of variance analysis was applied for determining the significant difference at $p<0.05$. The statistical analysis was conducted using SPSS 11 (SPSS Inc., Chicago, USA).

\section{Results and Discussion}

The sample information (Table 1) showed that paste with yolk-type (DYLP and SYLP) and paste-type (LP and BP) mooncakes accounted for $66 \%$, fruit flavor type (FF) and plant seed type (FK) accounted for $13 \%$ and $12 \%$, respectively, and other types including meat, cheese, and seafood type only accounted for $9 \%$ in Guangzhou mooncake market. Therefore, except for moisture, other parameters were not assayed for 9 other mooncakes.

3.1. Moisture. Moisture is an important parameter when considering mooncake quality because it significantly affects texture, taste, shelf life, and growth of the microbes. According to Chinese national standard of mooncake (GB 198552005), Guangdong-style mooncake should contain less than $25 \%$ moisture for paste type and fruit and vegetable type, less than $22.0 \%$ for yolk type and meat type and less than $19.0 \%$ for plant seed type, while Beijing-style should contain less than $17.0 \%$ moisture and Suzhou-style mooncake should contain less than $19.0 \%$ moisture for paste type, less than $12.0 \%$ for plant seed type, and less than $30.0 \%$ for meat type.

The average moisture content of the 100 samples value was $18.15 \%$. From the moisture frequency distribution (Figure 1), $87 \%$ of the samples contained $15.0 \sim 20.0 \%$ moisture and only $7 \%$ and $6 \%$ of samples contained less than $15.0 \%$ or higher than $21.0 \%$ moisture. The highest moisture was found in DYLP and BP mooncake (19.16\% and 19.12\%), followed by LP, SYLP, and FF mooncakes $(18.13 \%, 17.99 \%$, and $17.98 \%$, resp.). FK contained the least moisture (15.96\%). Except for FK type, the moisture content differences for DYLP, SYLP, LP, BP, and FF types were not significant $(p>0.05)$. Higher moisture content is one of the features of Guangdong-style mooncakes, giving them soft and oily properties. In order to keep the crispness of plant seeds, FK moon contained less moisture. 
TABLE 2: Firmness and cohesiveness of different types of Guangdong-style mooncakes.

\begin{tabular}{lcc}
\hline Mooncake type & Firmness $(\mathrm{N})$ & Cohesiveness \\
\hline DYLP & $148.91 \pm 63.62^{\mathrm{d}}$ & $2.93 \pm 1.84^{\mathrm{d}}$ \\
SYLP & $221.14 \pm 58.29^{\mathrm{c}}$ & $3.29 \pm 1.71^{\mathrm{c}}$ \\
LP & $219.76 \pm 65.00^{\mathrm{c}}$ & $3.34 \pm 1.78^{\mathrm{c}}$ \\
BP & $226.37 \pm 116.38^{\mathrm{c}}$ & $3.7 \pm 1.05^{\mathrm{b}}$ \\
FF & $268.55 \pm 114.54^{\mathrm{b}}$ & $3.43 \pm 1.88^{\mathrm{c}}$ \\
FK & $362.91 \pm 114.11^{\mathrm{a}}$ & $4.63 \pm 2.97^{\mathrm{a}}$ \\
\hline
\end{tabular}

Means without a common letter differ $(p>0.05)$

${ }^{*}$ DYLP: double-yolk lotus seed paste mooncake; SYLP: single-yolk lotus seed paste mooncake; LP: pure lotus seed paste mooncake; BP: bean paste mooncake; FF: fruit flavor mooncake; FK: five-kernel mooncake.

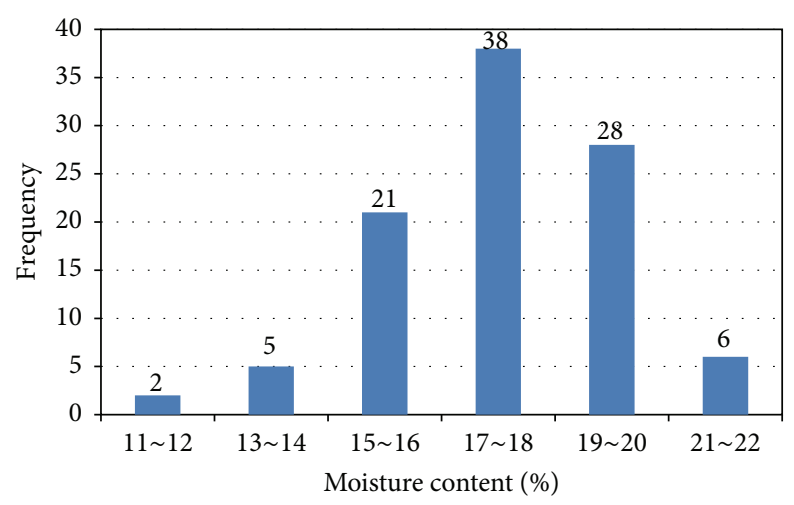

FIGURE 1: Moisture content (wet weight basis) frequency distribution of Guangdong-style mooncakes in the sample. Mean $=18.15 \%$, $\mathrm{SD}=2.15 \%$, and $n=100$.

3.2. Firmness and Cohesiveness. Studies have shown a relationship between mechanical testing (TPA) and the texture of food. TPA measures such parameters as chewiness, gumminess, cohesiveness, and firmness. Not only do these tests quantify the texture of the food, but it also evaluates the consistency of the manufacturing processes. Only firmness and cohesiveness were tested because Guangdongstyle mooncakes had high viscosity and poor fracturability, springiness, and resilience.

The firmness value is the peak force of the first compression of the product. The firmness need not occur at the point of deepest compression, although it typically does for most products. As shown in Table 2, the firmness of the different types of Guangdong-style mooncakes was in the following order: DYLP $<$ LP $<$ SYLP $<$ BP $<$ FF $<$ FK.

Cohesiveness is how well the product withstands a second deformation relative to how it behaved under the first deformation. It is measured as the area of work during the second compression divided by the area of work during the first compression. As shown in Table 2, the cohesiveness of different types of Guangdong-style mooncakes was in the following order: DYLP $<$ SYLP $<$ LP $<$ FF $<$ BP $<$ FK. Like the firmness, DYLP mooncakes had lowest cohesiveness (Table 2), while FK mooncakes had the highest cohesiveness (Table 2).
The food textures are highly related with their recipes and processes [7]. DYLP mooncake contains two yolks, while SYLP mooncake has one yolk in the filling. LP and BP mooncakes only have lotus paste or bean paste. Firmness of Guangdong-style mooncakes was negatively related to moisture content $(r=-0.910)$.

3.3. Reducing Sugar Content. Reducing sugar contents in Guangdong-style mooncakes were determined because they are highly related to blood glucose when people consume them. Average reducing sugar content in all tested samples was $37.57 \%$. As shown in Table 3, BP, LP, and FF mooncakes had higher reducing sugar, followed by SYLP and DYLP mooncakes, and FK mooncake had the lowest reducing sugar. Guangzhou Sibao SYLP and Hongkong Huangguan Winter Melon mooncakes had the highest reducing sugar $(51.48 \%$ and $48.17 \%$, resp.).

In Guangdong-style mooncakes, total sugar mainly comes from wheat flour, sucrose, and syrup, but total reducing sugar originates from the latter two resources. During manufacture of bean paste, lotus seed paste, and preserved fruit filling, large amounts of sucrose and syrup are added [13]. Owing to the relatively low sugar content in yolk, pork fat, and kernels, DYLP, SYLP, and FK mooncakes contained less total reducing sugar. However, even the least sugar containing mooncake (FK) still had $32.06 \%$ total reducing sugar. According to Chinese national standard (GB 198552005), total sugar in Guangdong-style mooncakes should be less than $46 \%, 45 \%, 42 \%, 38 \%, 38 \%$, and $36 \%$ in fruit and vegetable type, paste type, yolk type, plant seed type, meat type, and seafood type, respectively. Total sugar in Beijingstyle mooncake should be less than $40.0 \%$, while for Suzhoustyle mooncakes the level should be less than $38.0 \%$ for paste type, less than $27.0 \%$ for plant seed type, and less than $28.0 \%$ for meat type.

Mono- and disaccharides were also determined in Guangdong-style mooncake samples using HPLC; the result showed that sucrose, glucose, and fructose account for $77.30 \%, 10.98 \%$, and $8.77 \%$, respectively, while maltose and lactose account for less than $2.0 \%$ (detailed data not shown in this paper). Mono- and disaccharides contained in Guangdong-style mooncakes were highly related to the main carbohydrate materials, invert syrup, and sucrose.

Sugar is one of the most important ingredients used in bakery foods and plays a number of different roles in baking foods [14]. Sweetness is generally the most recognized functional property of sugar. Sugar can improve food shape and texture by playing a backbone role in baking foods. It can improve physical properties of dough by an antihydration action during mixing, regulating the degree of dough swelling, increasing plasticity, and providing good appearance and clear pattern for foods. Sugar with more moisture produces a soft and oily appearance just like in Guangdongstyle mooncakes. Sugar is an important contributor to flavor by interacting with other ingredients. Sugar is a good colorant of foods owing to its caramelization and Maillard reaction during baking can provide golden yellow and joyful baking flavor [15]. Caramelization results from the melting of sugars to create a deep brown color and new flavors. The Maillard 
TABle 3: Proximate composition of different types of Guangdong-style mooncakes.

\begin{tabular}{lcccccc}
\hline $\begin{array}{l}\text { Mooncake } \\
\text { type }\end{array}$ & $\begin{array}{c}\text { Moisture } \\
(\%)\end{array}$ & $\begin{array}{c}\text { Total reducing } \\
\text { sugar }(\%)\end{array}$ & $\begin{array}{c}\text { Crude fat } \\
(\%)\end{array}$ & $\begin{array}{c}\text { Protein } \\
(\%)\end{array}$ & $\begin{array}{c}\text { Cholesterol } \\
(\%)\end{array}$ & $\begin{array}{c}\text { Energy } \\
(\mathrm{kJ} / 100 \mathrm{~g})^{*}\end{array}$ \\
\hline DYLP & $19.16 \pm 1.61^{\mathrm{a}}$ & $35.15 \pm 4.40^{\mathrm{d}}$ & $18.74 \pm 2.88^{\mathrm{a}}$ & $6.61 \pm 0.30^{\mathrm{b}}$ & $1.89 \pm 0.75^{\mathrm{a}}$ & $1403.30 \pm 186.46^{\mathrm{a}}$ \\
SYLP & $17.99 \pm 1.90^{\mathrm{a}}$ & $37.75 \pm 3.77^{\mathrm{c}}$ & $14.80 \pm 3.04^{\mathrm{b}}$ & $6.56 \pm 0.42^{\mathrm{b}}$ & $0.93 \pm 0.44^{\mathrm{b}}$ & $1300.87 \pm 183.71^{\mathrm{b}}$ \\
LP & $18.13 \pm 1.59^{\mathrm{a}}$ & $41.53 \pm 2.29^{\mathrm{b}}$ & $9.86 \pm 2.73^{\mathrm{c}}$ & $6.32 \pm 0.97^{\mathrm{b}}$ & $0.22 \pm 0.13^{\mathrm{c}}$ & $1178.27 \pm 156.43^{\mathrm{c}}$ \\
BP & $19.12 \pm 2.21^{\mathrm{a}}$ & $43.12 \pm 3.44^{\mathrm{a}}$ & $10.46 \pm 1.30^{\mathrm{c}}$ & $6.74 \pm 0.58^{\mathrm{b}}$ & $0.07 \pm 0.03^{\mathrm{c}}$ & $1234.64 \pm 116.44^{\mathrm{c}}$ \\
FF & $17.98 \pm 1.59^{\mathrm{a}}$ & $41.29 \pm 4.86^{\mathrm{b}}$ & $3.72 \pm 1.17^{\mathrm{d}}$ & $5.35 \pm 0.26^{\mathrm{c}}$ & $0.15 \pm 0.03^{\mathrm{c}}$ & $930.52 \pm 130.33^{\mathrm{d}}$ \\
FK & $15.96 \pm 2.76^{\mathrm{b}}$ & $32.06 \pm 4.18^{\mathrm{e}}$ & $13.85 \pm 3.26^{\mathrm{b}}$ & $9.06 \pm 1.78^{\mathrm{a}}$ & $0.18 \pm 0.06^{\mathrm{c}}$ & $1211.49 \pm 221.94^{\mathrm{c}}$ \\
\hline
\end{tabular}

Note. ${ }^{*}$ The energy provided by starch was not calculated. Means without a common letter differ $(p>0.05)$. DYLP: double-yolk lotus seed paste mooncake; SYLP: single-yolk lotus seed paste mooncake; LP: pure lotus seed paste mooncake; BP: bean paste mooncake; FF: fruit flavor mooncake; FK: five-kernel mooncake.

reaction takes place when reducing sugars (e.g., glucose and fructose) and proteins are heated together. The yellow-brown colors that develop in baked foods can be attributed to the presence of sugar [16]. Sugar preserves more moisture for mooncakes by shortening baking time because of fast color forming during baking. Sugar acts as an important tenderizing agent in foods such as baked products [17]. Because sugar is a very important material in the production of mooncakes, people with diabetes mellitus should pay attention to this feature of mooncakes. Low sugar and sugarless mooncakes have surfaced to address health and lifestyle issues [18, 19]. These products are popular with diabetics, overweight consumers, or health and weight conscious consumers.

Sweetener sodium saccharine was determined in 91 Guangdong-style mooncake samples. The result showed that $15.52 \%$ of mooncakes contained this sweetener, mainly in FF mooncakes (55.56\%) and FK mooncakes (22.22\%), and the doses were less than that permitted in Chinese national standard $(0.15 \mathrm{~g} / \mathrm{kg})$. The result was consistent with literature on sodium saccharine used in mooncakes [20]. As other sweetening agents were not assayed in this research, they could not be excluded from the manufacture of mooncakes. The high content of total reducing sugar in Guangdong-style mooncakes decreased the need for using significant amounts of artificial synthetic sweeteners.

3.4. Fat, Fatty Acid Composition, and Cholesterol. Fat also plays an important role in cake manufacture, to entrap air during the creaming process, to physically interfere with the continuity of starch and protein particles, and to emulsify the liquid in formulation thus; fats contribute to the soft and tender eating properties required for cakes [21]. In mooncakes, fat also provides an oily mouth feel to the finished product.

Crude fat contents in Guangdong-style mooncakes were quantified using the Soxhlet extraction method. Except for meat and seafood mooncakes, average fat content in Guangdong-style mooncakes was $13.19 \%$. The DYLP mooncakes had the highest fat content, while FF mooncakes had the lowest fat content (Table 3). Fat contents in SYLP and FK mooncakes were similar, and difference in fat contents of pure LP and BP mooncakes was also not significant $(p>0.05)$.

In different types of Guangdong-style mooncakes, Mei Xin DYLP and Qu Xiang DYLP mooncakes were the highest
(26.01\%) and lowest (12.50\%) fat in DYLP type, respectively; Guangzhou Sibao SYLP and Guangzhou Jiujia SYLP mooncakes had the highest $(20.90 \%)$ and lowest $(8.47 \%)$ fat in SYLP type, respectively; Guangzhou Jiujia LP and Guangzhou Sibao LP had the highest (12.80\%) and lowest (4.24\%) fat in LP type, respectively; Tao Tao Ju FK and Hongkong Ronghua FK mooncakes had the highest (21.38\%) and lowest (8.66\%) fat in FK type, respectively; Tao Tao Ju BP and Hongkong Tangxin BP mooncake had the highest (11.59\%) and lowest (8.84\%) fat in BP type, respectively; FF mooncakes of different brands contained the low fat; especially Tian Xing Long FF had the least fat (1.90\%).

According to Chinese national standards, fat in Guangdong-style mooncake should be less than $18 \%$, $24 \%, 30 \%, 28 \%, 25 \%$, and $24 \%$ in fruit and vegetable type, paste type, yolk type, plant seed type, meat type, and seafood type, respectively. Fat in Beijing-style mooncake should be less than $20.0 \%$, while in Jiangsu-style mooncake should be less than $24.0 \%$ for paste type, less than $30.0 \%$ for plant seed type, and less than $33.0 \%$ for meat type. Fat in Guangdongstyle mooncakes mainly comes from vegetable oil, yolk, and plant seeds. Development of fruit flavor mooncakes significantly decreased the fat content in Guangdong-style mooncakes. It is good news for those people with high blood fat.

Fatty acid composition was analyzed by GC/MS method and confirmed by comparing retention times and MS Data (NIST 05a .L). Peaks with similarity higher than 95\% were confirmed as the fatty acid in the MS Data. The proportion of different fatty acids was calculated using the area normalization method (Table 4).

The most abundant fatty acids found in Guangdongstyle mooncakes were $\mathrm{C}_{18: 1}$ and $\mathrm{C}_{18: 2}$, which accounted for $73.10 \%$ of total fatty acids. The sum of unsaturated fatty acids in different Guangdong-style mooncake types was in the following order: FF $(87.53 \%)>$ BP $(78.20 \%)>$ SYLP $(76.46)$ $>$ FK $(74.78 \%)>$ LP $(74.36 \%)>$ DYLP $(70.45 \%)$. Part of fatty acids of yolk-type mooncakes (especially DYLP) was from salted yolk, so their unsaturated fatty acid ratios were relatively low. The fatty acid profile of LP mooncakes was similar to peanut oil [22-24], while that of FF and BP was similar to soybean oil $[25,26]$. Traditionally, vegetable oil used in Guangdong-style mooncake manufacture is peanut oil, but soybean oil and corn oil are also used by many 


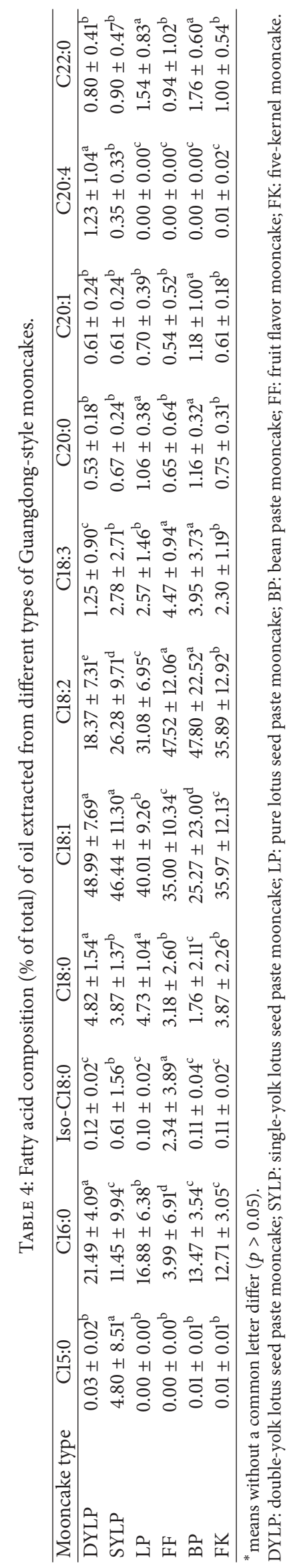


factories in order to decrease costs. Vegetable oil types used in DYLP, SYLP, and FF mooncakes could not be speculated by fatty acid profile which would be affected by fatty acids from yolk or plant seeds. Salted duck yolk contained approximately $50 \%$ lipid and its fatty acid profile was quite different from vegetable oil based mooncakes. Main fatty acids in salted duck yolk were $\mathrm{C}_{16: 0}(27.5 \%), \mathrm{C}_{16: 1 \mathrm{n}}(2.66 \%), \mathrm{C}_{18: 0}(5.63 \%)$, $\mathrm{C}_{18: \ln }(48.40 \%), \mathrm{C}_{18: 2 \mathrm{n}}(6.88 \%)$, and $\mathrm{C}_{20: 4 \mathrm{n}}(2.79)$ [27]. DYLP and SYLP mooncakes embodied two- and one-salted duck yolk, respectively. Therefore, the salted yolk fat had great influence on the yolk-type mooncake fatty acid profiles.

Cholesterol content was quantified by ferric ammonium sulfate color reagent method and the results were shown in Table 3. Average cholesterol content was $0.76 \%$ in all tested Guangdong-style mooncake samples. Cholesterol contents in DYLP, SYLP, LP, FK, BP, and FF mooncakes were $1.89 \%$, $0.93 \%, 0.22 \%, 0.18 \%, 0.15 \%$, and $0.07 \%$, respectively, which were positively correlated with their crude fat contents $(r=$ 0.798 ). It was reported that cholesterol content in duck eggs was $10.36 \mathrm{mg} / \mathrm{g}$ or $13.15 \mathrm{mg} / \mathrm{g}$ of yolk [28, 29]. It could be boldly speculated that most of the cholesterol came from yolks because only yolk types contained high contents and DYLP mooncake had double the content of SYLP mooncakes.

3.5. Protein Content. The protein contents of Guangdongstyle mooncakes were determined by the macro-Kjeldahl method and the results were shown in Table 3. The result showed that protein content in Guangdong-style mooncakes was from $4.67 \%$ to $10.31 \%$, FK type contained the highest protein, and there was no difference among the other five types. Protein in Guangdong-style mooncakes mainly comes from wheat flour, albumen, bean, lotus seed, and other plant seeds. Plant seeds in FK mooncake filling make it rich in protein.

In Guangdong-style mooncakes, protein is not an important index for the quality control. There is no protein criterion in quality standards of yolk-type, fruit-type, and pastetype mooncakes in Chinese national standards. The protein content requirement for plant seed type and meat type should be more than $5.5 \%$ and for seafood type should be more than $5.0 \%$.

3.6. Energy. The caloric values of different types of Guangdong-style mooncakes were calculated by using the Atwater energy conversion factors and the results were shown in Table 3. The results indicated that Guangdong-style mooncakes could provide $930.52-1403.30 \mathrm{~kJ} / 100 \mathrm{~g}$ calories, and DYLP mooncakes contained the highest energy while FF mooncakes had the least energy in tested six types of Guangdong-style mooncakes. LP, BP, and FK mooncakes contained similar calories, and there was no significant difference between SYLP and BP mooncakes in energy content $(p>0.05)$.

From the literature, potential beneficial effects of intake of starchy foods, especially those containing slowly digestible and resistant starches, and potential detrimental effects of high intakes of fructose become apparent [30]. An intake of whole grains, legumes, and vegetables, which contain more appropriate sources of carbohydrates, is associated with reduced risk of cardiovascular and other chronic diseases, rather than foods rich in sugars, especially in the form of sugar-sweetened beverages [30]. Due to similar ingredients but high moisture content, Guangdong-style mooncakes provide less energy than most biscuits [31].

In recent years, to adapt to increasing health-conscious lifestyle, low calories and fat-free mooncakes have also appeared. Some are made of yogurt, jelly, and fat-free icecream. Even high-fiber low-sugar mooncakes have made their appearance [32].

\section{Conclusions}

Guangdong-style mooncakes contained 15.96 19.16\% moisture. Higher moisture content is one of the features of Guangdong-style mooncake and gives it soft and oily properties. Guangdong-style mooncakes contain high level of reducing sugar, mainly sucrose, glucose, and fructose. There was a big difference in the fat contents in Guangdongstyle mooncakes. DYLP mooncakes contained $18.74 \%$, while FF only contained $3.72 \%$ of fat. Dominating fatty acids in Guangdong-style mooncakes were $\mathrm{C}_{18: 1}$ and $\mathrm{C}_{18: 2}$. Cholesterol contents in Guangdong-style mooncakes were positively correlated with their crude fat contents. Protein content in Guangdong-style mooncakes was from $4.67 \%$ to $10.31 \%$. Guangdong-style mooncakes could provide $930.52-$ $1403.30 \mathrm{~kJ} / 100 \mathrm{~g}$ calories. Guangdong-style mooncakes are baking foods high in moisture and sugar, but not always high in fat. These findings are of importance to develop healthier nutritional mooncakes while helping consumer's selection of these foods according to their health conditions. In order to satisfy consumers with increasing health consciousness, new mooncakes with low sugar, low fat, and low calories but rich in functional components should be developed in the future.

\section{Conflict of Interests}

The authors declare that there is no conflict of interests regarding the publication of this paper.

\section{Acknowledgments}

This work was financially supported by Integrated Project of Industry, Education and Research of Guangdong Province and Ministry of Education, China (2012B091000127), and Improving Science and Technology Service Development Projects of Guangdong Province (2013B040400009, 2014B040404059).

\section{References}

[1] C. S. Tang, K. Rajaram, A. Alptekinoğlu, and J. Ou, "The benefits of advance booking discount programs: model and analysis," Management Science, vol. 50, no. 4, pp. 465-478, 2004.

[2] C. S. Tang, K. Rajaram, and J. H. Ou, "Managing demand uncertainty for short life cycle products using advance booking discount programs," in Supply Chain Management: Models, Applications, and Research Directions, vol. 62 of Applied Optimization, pp. 69-95, Springer, New York, NY, USA, 2005. 
[3] K. J. S. Bergquist, "From Kim Chee to moon cakes: feeding Asian adoptees' imaginings of culture and self," Food, Culture and Society, vol. 9, no. 2, pp. 141-153, 2006.

[4] A. K. K. Chan, L. T. Denton, and A. S. L. Tsang, "The art of gift giving in China," Business Horizons, vol. 46, no. 4, pp. 47-52, 2003.

[5] T. Chysirichote, A. Utaipatanacheep, and W. Varanyanond, "Effect of reducing fat and using fat replacers in the crust of flaky Chinese pastry," Kasetsart Journal (Natural Science), vol. 45, pp. 120-127, 2011.

[6] C. L. Jia, Y. S. Kim, W. N. Huang, and G. W. Huang, "Sensory and instrumental assessment of Chinese moon cake: Influences of almond flour, maltitol syrup, fat, and gums," Food Research International, vol. 41, no. 9, pp. 930-936, 2008.

[7] B. Wen, Z. C. Wei, M. W. Zhang, Y. Zhang, Y. Y. Deng, and $\mathrm{X}$. J. Tang, "Correlation between sensory evaluation and TPA parameters of Guangdong-style moon cake," Journal of the Chinese Cereals and Oils Association, vol. 27, no. 1, pp. 91-96, 2012 (Chinese).

[8] M. Gómez, B. Oliete, C. M. Rosell, V. Pando, and E. Fernández, "Studies on cake quality made of wheat-chickpea flour blends," LWT: Food Science and Technology, vol. 41, no. 9, pp. 1701-1709, 2008.

[9] AOAC, Official Methods of Analysis, AOAC, Washington, DC, USA, 17th edition, 2000.

[10] J. V. O'Fallon, J. R. Busboom, M. L. Nelson, and C. T. Gaskins, “A direct method for fatty acid methyl ester synthesis: application to wet meat tissues, oils, and feedstuffs," Journal of Animal Science, vol. 85, no. 6, pp. 1511-1521, 2007.

[11] H. W. Feng, C. M. Pi, R. B. Wang, and L. C. Chen, "Use of ferric ammonium sulfate in serum cholesterol determination," Clinical Chemistry, vol. 19, no. 1, pp. 121-122, 1973.

[12] G. Piñeiro-Avila, A. Salvador, and M. De La Guardia, "Microwave-assisted saponification of animal greases for cholesterol determination," Analytica Chimica Acta, vol. 371, no. 2-3, pp. 297-303, 1998.

[13] W. H. Zhao, W. D. Bai, X. J. Yang, and M. Qian, "Chemical change of the Cantonese moon cake in storage period," Academic Periodical of Farm Products Processing, no. 6, pp. 14-17, 2009 (Chinese).

[14] B. Pareyt, F. Talhaoui, G. Kerckhofs et al., "The role of sugar and fat in sugar-snap cookies: structural and textural properties," Journal of Food Engineering, vol. 90, no. 3, pp. 400-408, 2009.

[15] H. T. Nguyen, H. J. Van der Fels-Klerx, R. J. B. Peters, and M. A. J. S. Van Boekel, "Acrlamide and 5-hydroxymethylfurfural formation during baking of biscuits: part I; effects of sugar type," Food Chemistry, vol. 192, pp. 575-585, 2016.

[16] E. Purlis, "Browning development in bakery products -a review," Journal of Food Engineering, vol. 99, pp. 239-249, 2010.

[17] J. Gusba, "Sugar, sugar!: A look at the functional role of sugar in baking," 2008, http://www.bakersjournal.com/technical/sugarsugar-a-look-at-the-functional-role-of-sugar-in-baking-967.

[18] J. Z. Meng, Y. Q. Wei, and X. M. Qin, "Study on moon cake filling prepared with trehalose," Food Science, vol. 27, no. 7, pp. 273275, 2006.

[19] Y. Zhang, J. W. Chi, X. J. Tang, and Z. C. Wei, "Development of pumpkin mooncake," Food and Nutrition in China, no. 2, pp. 44-46, 2008 (Chinese).

[20] S. H. Cheng, L. Ding, L. Lin, and C. L. Yang, "Simultaneous determination of benzoic acid, sorbic acid and saccharin sodium in moon cake products by high performance liquid chromatography," Food Science, vol. 29, no. 6, pp. 376-378, 2008.
[21] M. Sowmya, T. Jeyarani, R. Jyotsna, and D. Indrani, "Effect of replacement of fat with sesame oil and additives on rheological, microstructural, quality characteristics and fatty acid profile of cakes," Food Hydrocolloids, vol. 23, no. 7, pp. 1827-1836, 2009.

[22] T. H. Sanders, "Fatty acid composition of lipid classes in oils from peanuts differing in variety and maturity," Journal of the American Oil Chemists' Society, vol. 57, no. 1, pp. 12-15, 1980.

[23] P. C. Andersen, K. Hill, D. W. Gorbet, and B. V. Brodbeck, "Fatty acid and amino acid profiles of selected peanut cultivars and breeding lines," Journal of Food Composition and Analysis, vol. 11, no. 2, pp. 100-111, 1998.

[24] C. Y. Yang, X. M. Liu, and Z. Y. Chen, "Determination of fatty acid profiles in fifteen kinds of edible vegetable oil by gas chromatography-mass spectrometry," Journal of Food Science, vol. 34, no. 6, pp. 211-214, 2013 (Chinese).

[25] B. M. Craig and N. L. Murty, "Quantitative fatty acid analysis of vegetable oils by gas-liquid chromatography," Journal of the American Oil Chemists Society, vol. 36, no. 11, pp. 549-552, 1959.

[26] A. Kamal-Eldin and R. Andersson, "A multivariate study of the correlation between tocopherol content and fatty acid composition in vegetable oils," Journal of the American Oil Chemists' Society, vol. 74, no. 4, pp. 375-380, 1997.

[27] T. Kaewmanee, S. Benjakul, and W. Visessanguan, "Changes in chemical composition, physical properties and microstructure of duck egg as influenced by salting," Food Chemistry, vol. 112, no. 3, pp. 560-569, 2009.

[28] Z. Aziz, S. Cyriac, V. Beena, and P. T. Philomina, "Comparison of cholesterol content in chicken, duck and quail eggs," Journal of Veterinary and Animal Sciences, vol. 43, pp. 64-66, 2012.

[29] D. X. Huang, J. B. Li, H. W. Chen, Y. L. He, J. B. Xu, and F. L. Chen, "Comparison of the cholesterol content in hen eggs, duck eggs and quail eggs," Anhui Agricultural Science Bulletin, vol. 19, no. 11, pp. 109-110, 2013 (Chinese).

[30] E. E. J. G. Aller, I. Abete, A. Astrup, J. A. Martinez, and M. A. van Baak, "Starches, sugars and obesity," Nutrients, vol. 3, no. 3, pp. 341-369, 2011.

[31] M. Roe, S. Church, and P. Finglas, "Nutrient analysis survey of biscuits, buns, cakes and pastries," Analytical Report, 2011, https://www.gov.uk/government/uploads/system/uploads/ attachment_data/file/216389/dh_129729.pdf.

[32] X. L. Li and L. X. Huang, "Manufacture of vegetable and fruit moon cake stuffing with low sugar," Food Science and Technology, vol. 4, pp. 106-107, 2007 (Chinese). 

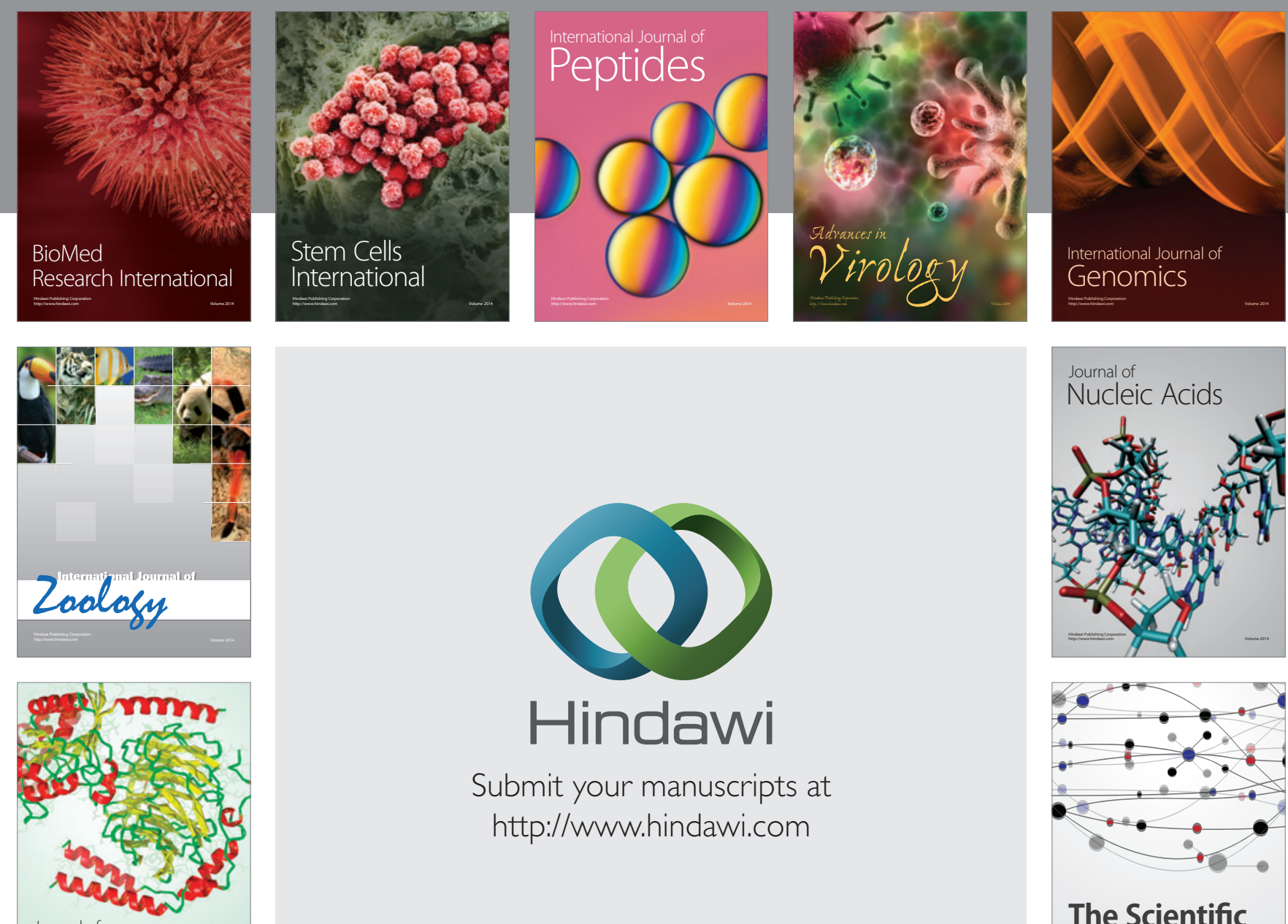

Submit your manuscripts at

http://www.hindawi.com

Journal of
Signal Transduction
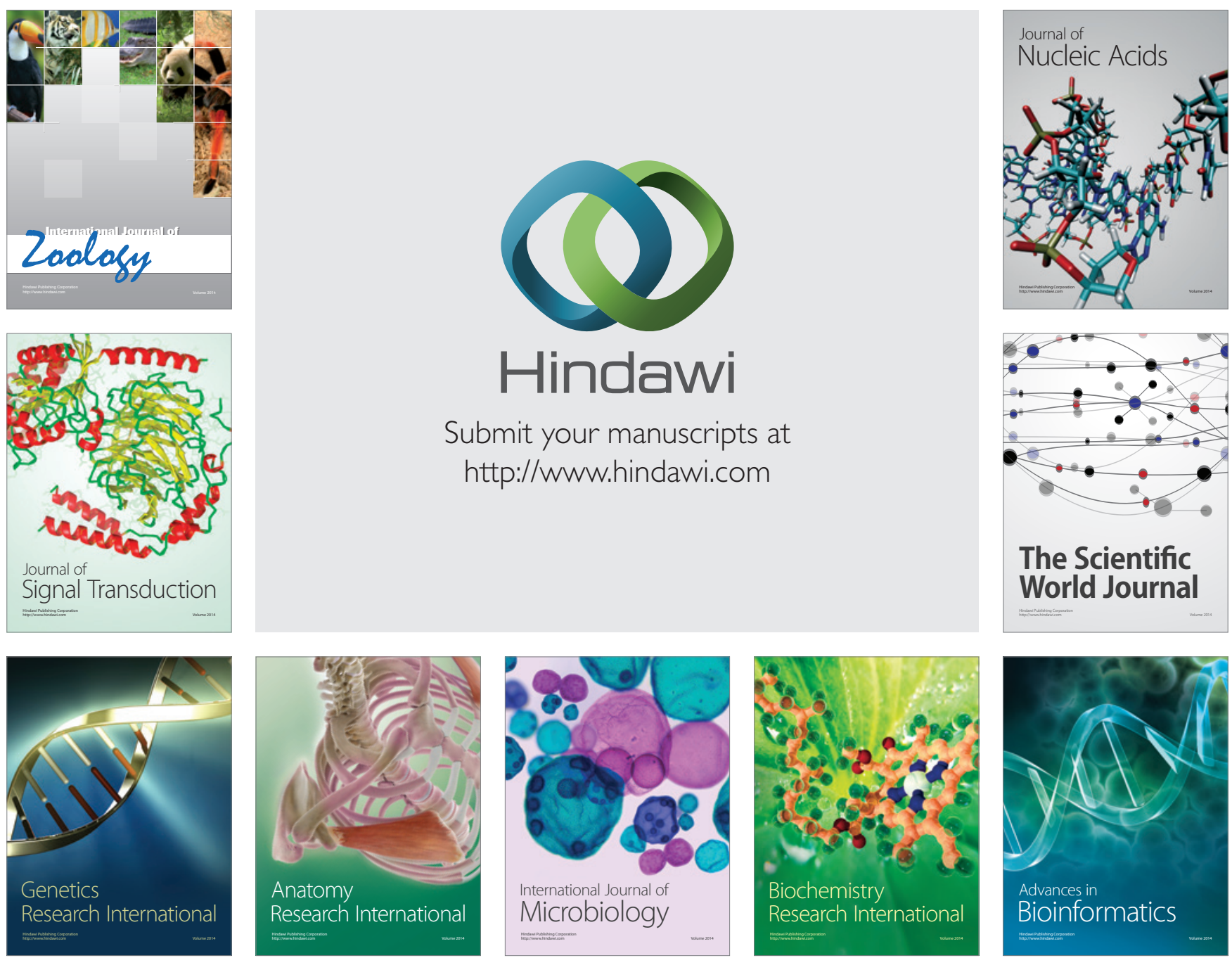

The Scientific World Journal
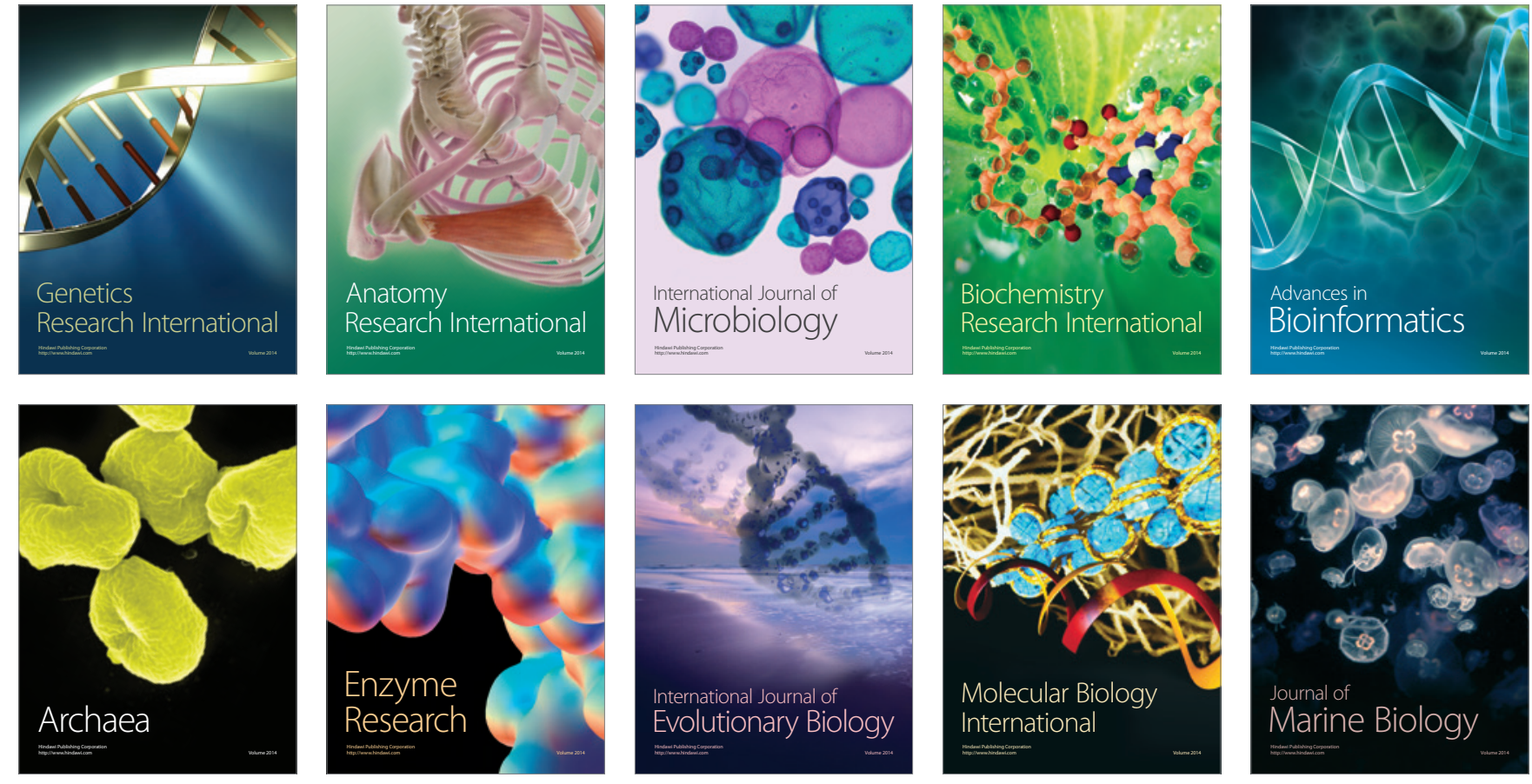